山्山FFRANÇAISE

$>\mathrm{DE}$

$\stackrel{1=1}{\simeq}$ PÉDAGOGIE

\section{Revue française de pédagogie}

Recherches en éducation

$186 \mid 2014$

Les trajectoires des inspections scolaires en Europe : analyses comparatives

\title{
Penser l'égalité professionnelle entre les hommes et les femmes dans l'enseignement du second degré : une comparaison France-Angleterre
}

Thinking gender equality in the secondary school teaching profession: A

comparison between France and England

\section{Marie-Pierre Moreau}

\section{OpenEdition}

Journals

Édition électronique

URL : http://journals.openedition.org/rfp/4425

DOI : $10.4000 /$ rfp. 4425

ISSN : 2105-2913

Éditeur

ENS Éditions

Édition imprimée

Date de publication : 1 janvier 2014

Pagination : $99-113$

ISBN : 978-2-84788-639-9

ISSN : 0556-7807

Référence électronique

Marie-Pierre Moreau, «Penser l'égalité professionnelle entre les hommes et les femmes dans l'enseignement du second degré : une comparaison France-Angleterre », Revue française de pédagogie [En ligne], 186 | 2014, mis en ligne le 01 janvier 2017, consulté le 20 avril 2019. URL : http:// journals.openedition.org/rfp/4425; DOI : 10.4000/rfp.4425 


\title{
Penser l'égalité professionnelle entre les hommes et les femmes dans l'enseignement du second degré : une comparaison France-Angleterre
}

\author{
Marie-Pierre Moreau
}

À partir d'une analyse comparative France-Angleterre et d'une architecture conceptuelle empruntant aux théories des rapports sociaux de sexe et à la sociologie du travail et de l'éducation, cet article questionne la représentation de l'enseignement du second degré comme profession qui offrirait des opportunités professionnelles similaires aux hommes et aux femmes qui l'exercent. Les statistiques nationales et l'analyse d'un corpus d'entretiens permettent de mettre au jour les formes de la différentiation sexuée des carrières enseignantes, selon des modalités toutefois spécifiques à chaque contexte sociétal qui sont fonction des politiques publiques et des cultures professionnelles en place.

Mots-clés (TESE) : genre, enseignant, égalité des sexes, France, Angleterre.

\section{INTRODUCTION}

Dans la plupart des sociétés modernes, le professorat constitue un des principaux débouchés professionnels pour les femmes. Pour autant, peu de sociologues ont étudié les trajectoires professionnelles enseignantes à partir de la perspective des rapports sociaux de sexe. La relative invisibilité de cette question sur un plan sociologique et politique (Moreau, 2011b) aurait trait à une conception de l'enseignement comme d'un métier offrant des opportunités similaires aux hommes et aux femmes, voire comme «bien... pour une femme», selon le titre (critique) d'un article de M. Cacouault (1987). Cette conception dissimule plusieurs présupposés. En France et en Angleterre, comme dans de nombreux autres pays, la mixité relative de l'enseignement du second degré par rapport à des ratios hommes/femmes très différents dans le premier degré et dans l'enseignement supérieur peut amener à conclure hâtivement à l'égalité professionnelle entre les hommes et les femmes à ce niveau du système éducatif. Par ailleurs, les conditions d'exercice de ce métier sont souvent perçues comme favorisant l'articulation vie professionnelle-vie familiale, elle-même perçue comme une «affaire de femmes» (Delphy, 1993). Enfin, 
la majorité des enseignants du second degré bénéficient du statut d'employé du secteur public, alors même que les règles de recrutement et de promotion qui prévalent dans ce secteur sont généralement perçues comme le garant de l'égalité professionnelle (Fortino, 2002). Cette conception de l'enseignement du second degré est fort répandue, y compris parmi ceux et celles qui exercent ce métier (Cacouault, 2003; Chan, Ngai \& Choi, 2014; Moreau, Osgood \& Halsall, 2007; Moreau, 2014).

Sans revenir ici de manière détaillée sur l'évolution socio-historique de ce métier, on peut rappeler que l'ouverture de l'enseignement aux femmes comme débouché professionnel et sur une grande échelle est relativement récente. En Angleterre, ce n'est qu'en 1944 que le marriage bar, qui obligeait les femmes à quitter certaines professions lorsqu'elles se mariaient, a été aboli. Aujourd'hui, en Angleterre comme dans un grand nombre de pays, ces interdictions légales ont cessé d'exister. Mais, si l'enseignement s'avère moins pénalisant pour les femmes que d'autres secteurs d'activité (INSEE, 2011), peut-on pour autant parler d'une profession offrant des opportunités de carrière similaires aux hommes et aux femmes? Par ailleurs, si inégalités de sexe il y a, comment s'élaborent-elles et quel rôle jouent dans ce processus les politiques publiques et les cultures professionnelles enseignantes?

Afin de répondre à ces questions, nous nous appuyons sur les résultats d'une étude portant de manière plus générale sur l'influence des rapports sociaux de sexe sur les trajectoires professionnelles et familiales des enseignants français et anglais du second degré (Moreau, 2011a), en recentrant dans cet article l'analyse sur la question de l'égalité professionnelle. Après avoir présenté le cadre théorique et la méthodologie, nous procédons à un rapide retour sur certains des principes qui animent les États-providence et les cultures professionnelles enseignantes dans les deux pays considérés. Nous examinons ensuite les caractéristiques spécifiques de la différentiation sexuée des carrières enseignantes dans ces deux contextes, avant d'analyser la manière dont les politiques publiques et les cultures professionnelles contribuent à la production des inégalités professionnelles.

\section{CADRE THÉORIQUE ET MÉTHODOLOGIE}

Cette contribution prend appui sur la perspective des sociologues du travail et de l'éducation intégrant les théories des rapports sociaux de sexe, qui "pose comme centrale la problématique de la domination entre les sexes et ses conséquences " (Hirata, Laborie, Le Doare et al., 2000, p. IX) et définit le genre comme « un système social de différentiation et de hiérarchisation " (Le Feuvre, 2002, p. 12). Sur un plan conceptuel, ces travaux ont montré comment l'assignation prioritaire des femmes au domestique et au care a engendré leur subordination professionnelle (Chadeau \& Fouquet, 1981; Collectif, 1984) et apporté un éclairage nouveau sur la manière dont des modes de fonctionnement organisationnels a priori neutres produisent de la différentiation sexuée. Ce cadre d'analyse a donné lieu à de nombreux travaux sur des professions diverses (par exemple Fave-Bonnet, 1999; Fouquet \& Laufer, 2001; Le Feuvre, Lapeyre, Cacouault et al., 2003), allant au-delà du seul constat de l'exclusion ou de la sous-représentation des femmes dans les positions hiérarchiques les plus élevées, afin d'explorer plus finement les formes de la division sexuelle du travail salarié (Buscatto \& Marry, 2009). Certains de ces travaux ont ainsi montré que la mixité d'une profession (au sens d'une co-présence des hommes et des femmes dans des proportions significatives) ne garantit pas l'égalité professionnelle (Fortino, 2002) et que les hommes tendent à bénéficier de carrières accélérées, y compris dans les professions où ils sont minoritaires (Williams, 1992). Ces recherches ont cependant rarement porté sur les professions «mixtes» ou dans lesquelles les femmes sont majoritaires, un constat qui vaut aussi pour l'enseignement du second degré comme on le notait déjà en introduction. S'il existe des exceptions, les travaux disponibles ont tendance à porter uniquement sur les femmes ou sur l'accès aux postes de direction (voir, par exemple, Cacouault, 1987; Cacouault-Bitaud, 2007; Coleman, 2002).

La comparaison internationale représente ici un outil méthodologique et heuristique qui permet, par effet de contraste, de saisir les logiques sociétales à l'œuvre lors de la production des inégalités de genre. Alors que le "masculin » et le "féminin » font communément l'objet d'un processus de naturalisation (Guillaumin, 1992), l'exercice comparatif met au jour la variabilité des normes de sexe. L'approche comparative proposée reprend certains des principes de l'analyse sociétale (Maurice, 1989; Maurice, Sellier \& Sylvestre, 1982, 1992), notamment l'identification de "cohérences sociétales " et la contextualisation des phénomènes comparés, qui offre une alternative aux postulats fonctionnaliste (présupposant l'universalité des faits comparés et l'existence d'équivalents stricts) et culturaliste (qui, à trop vouloir tenir compte des spécificités culturelles, complique l'exercice comparatif). Mais l'approche retenue tient aussi compte de 
certaines des critiques dont a fait l'objet l'analyse sociétale (Lallement \& Spurk, 2003), notamment pour avoir minimisé les phénomènes de différentiation interne propres à chaque contexte national, y compris du point de vue des positions sociales occupées par les hommes et les femmes (Marry, 1998; O'Reilly, 2000). II s'agit donc de privilégier une analyse qui, plutôt que de construire les territoires comparés comme des entités relativement homogènes et stables, articule plusieurs niveaux de la réalité sociale, soit les niveaux sociétal ou national, mésosocial (dans ce cas, sectoriel) et individuel, sur le modèle des travaux comparatifs de sociologues du travail s'appuyant sur la perspective théorique des rapports sociaux de sexe (Crompton, 1999; Le Feuvre, 2008).

L'appréhension de ce dernier niveau d'analyse (celui des biographies individuelles) est favorisée par une méthodologie s'appuyant principalement sur la conduite d'entretiens de type semi-directif, un format suffisamment flexible pour saisir la parole et l'expérience de chaque interviewé dans sa singularité et suffisamment structuré pour rendre la comparaison possible. L'article s'appuie sur soixante entretiens, qui ont été menés par l'auteur avec des enseignants d'établissements anglais et français du second degré. La population d'enquête était composée en proportions égales d'hommes et de femmes, titulaires en France de l'agrégation, du Certificat d'aptitude au professorat de l'enseignement secondaire (CAPES) ou équivalent, et en Angleterre du Qualified Teacher Status, une accréditation obtenue à l'issue de la formation initiale. Tous les interviewés, sauf un, exerçaient dans un établissement public au moment de l'entretien. La plupart avaient entre 35 et 45 ans, un groupe d'âge qui correspond généralement dans ces deux pays au milieu de carrière. Les entretiens ont été conduits la plupart du temps dans l'établissement, plus rarement au domicile de l'interviewé ou dans un lieu public, selon les disponibilités et les préférences des participants. En dehors des critères d'âge et de sexe, le recrutement de la population d'enquête a répondu à une logique de diversification du point de vue de variables perçues comme étant a priori susceptibles d'exercer une influence sur les expériences professionnelles : la zone géographique, la qualification et le type de poste, le type d'établissement, la discipline enseignée et la situation familiale. Les entretiens ont été retranscrits dans leur totalité et, suite à des lectures répétées, ont donné lieu à l'élaboration d'une grille d'analyse thématique (Robson, 1993). Des thèmes dérivant des hypothèses ont été identifiés, chaque entretien étant ensuite soumis à ce découpage thématique.

\section{ÉTATS-PROVIDENCE, NORMES DE L'ACTIVITÉ SALARIÉE ET CULTURES SCOLAIRES : ÉLÉMENTS DE CONTEXTUALISATION}

La France et l'Angleterre offrent ici un contraste intéressant du point de vue de l'intervention des politiques publiques dans la gestion de l'articulation vie familialevie professionnelle. En France, les politiques publiques sont plutôt marquées par des principes d'inspiration familialiste mais aussi égalitaire (Daune-Richard, 1999), ainsi que par une intervention dans la vie privée des citoyens généralement perçue comme légitime (Esping-Andersen, 1990). L'activité salariée des femmes et leur natalité sont encouragées, bien que certaines mesures aient parfois incité à leur retrait du marché du travail (Martin, 1998). En comparaison, les politiques publiques anglaises se caractérisent par une orientation plutôt néo-libérale et individualiste (Faucher-King \& Le Galès, 2010). La gestion de l'articulation vie familiale-vie professionnelle est perçue comme relevant principalement de la responsabilité des acteurs, la parentalité étant appréhendée avant tout comme un choix individuel (Gregory \& Windebank, 2000). II n'existe pas dans ce pays d'écoles maternelles, et le coût des structures de garde d'enfants est élevé pour les familles, bien que l'État ait progressivement investi ce champ depuis les années 1990.

L'Angleterre et la France offrent aussi un contraste intéressant du point de vue de leurs systèmes scolaires et des cultures professionnelles enseignantes qui prévalent dans chacun de ces pays. Sans revenir ici de manière détaillée sur les caractéristiques des systèmes éducatifs français et anglais, on rappellera que le système scolaire français a été historiquement marqué du sceau de l'idéal républicain d'une école pour tous. En comparaison, la politique dominante des gouvernements britanniques vise principalement depuis plusieurs décennies la diversification de l'offre scolaire et l'application des principes du marché au secteur éducatif (Faucher-King \& Le Galès, 2010). Suite à l'introduction de l'Education Reform Act de 1988, le pouvoir des autorités locales a été considérablement affaibli, avec le transfert du processus décisionnel aux conseils d'administration des établissements scolaires et à l'État central. Dans un contexte décrit comme celui d'un quasi-marché (Glennerster, 1991), les établissements se trouvent toujours plus en situation de concurrence.

Contrairement à leurs homologues français, les enseignants anglais sont recrutés suite à des formations privilégiant la pratique sur l'expertise disciplinaire. II n'existe 
pas en Angleterre d'épreuve finale qualifiante et, à l'issue de leur formation, les enseignants doivent candidater auprès des directions d'établissement et conseils d'administration qui gèrent et recrutent la main-d'œuvre pour obtenir un emploi. Comme nous l'avons montré ailleurs, les motivations mises en avant par les enseignants et les identités professionnelles enseignantes sont profondément marquées par le contexte sociétal (Moreau, 2014). En France, l'expertise disciplinaire constitue un ressort identitaire essentiel et une motivation importante dans le choix de ce métier, bien que cette conception de l'enseignant comme transmetteur de savoirs disciplinaires soit de moins en moins tenable face à l'hétérogénéité des publics scolaires et à l'élargissement progressif du rôle des enseignants (Moreau, 2013). En Angleterre, le travail auprès des enfants et notamment l'aide à la formation d'un «individu complet sous toutes ses facettes » (le well-rounded individual) constituent une motivation importante dans le choix de cette profession (Raveaud, 2006). Les attentes à l'égard des enseignants sont aussi beaucoup plus vastes par rapport à ce que l'on observe en France. La gestion des ressources, administratives et financières, de l'établissement, ou encore le soin à l'enfant dans ses aspects sociaux et psychologiques (pastoral care) sont perçus par tous comme des aspects légitimes de ce métier (Moreau, 2013). Mais force est de constater que, dans un contexte caractérisé par la mise en concurrence toujours plus forte des établissements sur la base des résultats scolaires, le métier d'enseignant consiste de plus en plus à améliorer la performance des élèves (Mahony \& Hextall, 2000; Malet \& Brisard, 2005).

Enfin, un autre contraste entre ces deux pays concerne les caractéristiques spatio-temporelles associées à l'exercice de ce métier. L'obligation de présence dans l'établissement des enseignants français du second degré se limite pour l'essentiel aux heures d'enseignement, précisées par le décret du 25 mai 1950 (15 heures hebdomadaires pour un agrégé, 18 heures pour un certifié, avec des modulations dépendant de nombreux facteurs). La flexibilité spatio-temporelle de ce métier tend cependant à s'amoindrir, du fait de responsabilités de plus en plus vastes et de pratiques de concertation toujours plus nombreuses. En Angleterre, suite au Teachers Pay and Conditions Act de 1987, les enseignants du second degré sont tenus de travailler un minimum de 1265 heures dans l'établissement, étalées sur 195 jours, soit en moyenne 6 heures 30 de présence quotidienne, qui ne couvrent pas le temps consacré à la préparation des cours, à la correction de copies et à l'écriture de rapports sur les élèves. Les directions des établissements peuvent cependant exiger des enseignants qu'ils accom- plissent un plus grand nombre d'heures si nécessaire à la réalisation de leurs missions, sans majoration de salaire.

\section{LES FORMES DE LA DIFFÉRENTIATION SEXUÉE DES CARRIÈRES ENSEIGNANTES EN FRANCE ET EN ANGLETERRE}

Dans chacun des pays considérés, le taux de féminisation de cette profession au niveau national varie considérablement selon le cycle et le type d'établissement (Department for Education, 2013; Ministère de l'Éducation nationale, 2013) ${ }^{1}$. En France, en 2012-2013, les femmes représentaient $58 \%$ des enseignants du second degré $(63 \%$ en collège et $53 \%$ en lycée d'enseignement général et technologique). Certains travaux montrent aussi que la part des hommes est plus élevée encore dans les établissements prestigieux, dans les sections post-baccalauréat des lycées et parmi ceux qui y effectuent l'ensemble de leur service (Cacouault-Bitaud, 2007; Léger, 1983). En Angleterre, en 2012-2013, les femmes représentaient $61 \%$ des enseignants du second degré. II faut cependant rappeler que ce taux de féminisation est en réalité plus élevé, puisque les enseignants à temps partiel, ainsi d'ailleurs que les enseignants non qualifiés et «remplaçants» ne sont pas inclus dans ces chiffres, alors même qu'une très large proportion sont des femmes (Hutchings, Smart, James et al., 2006).

En France, où domine une identité professionnelle d'expert disciplinaire parmi ce groupe (Moreau, 2011a), l'enseignement à des classes au niveau avancé dans la discipline enseignée (classes de lycée, classes à examen et classes préparatoires aux grandes écoles) et l'obtention de l'agrégation, qualification la plus élevée validant l'expertise disciplinaire, sont associés à un fort prestige et, dans certains cas, à un surcroît de salaire. En 2012-2013, les femmes représentaient $62 \%$ des certifiés, mais $51 \%$ des agrégés et $32 \%$ des professeurs de chaire supérieure (Ministère de l'Éducation nationale, 2013). En Angleterre, il n'existe pas de distinction équivalente à celle observée en France entre le corps des agrégés et celui des certifiés. Mais on observe dans ce pays aussi une sous-représentation des femmes dans les postes les plus élevés de la hiérarchie interne du métier. Une identité professionnelle de type «managérial » domine, dans un contexte où la progression de carrière se traduit principalement par l'accès à des postes associés avec des responsabilités accrues en matière de gestion des res- 
sources humaines, financières et administratives. Or, les femmes tendent à être sous-représentées dans les postes de management. En 2012-2013, elles représentaient $63 \%$ des classroom teachers des établissements anglais du second degré, $49 \%$ des deputy/assistant headteachers et $36 \%$ des headteachers (Department for Education, 2013). Les résultats d'une enquête plus ancienne menée auprès d'un large échantillon et incluant les enseignants exerçant à temps partiel suggèrent que les femmes représenteraient en réalité $77 \%$ des classroom teachers (Hutchings, Smart, James et al., 2006).

Les récits des enseignants interviewés dans le cadre de notre enquête mettent au jour l'existence de différences importantes entre les deux pays considérés, mais aussi, au sein de chaque pays, entre les hommes et les femmes du point de vue des normes de l'activité professionnelle. En France comme en Angleterre, les enseignants sans enfant ont des modalités de carrière relativement similaires. Mais, lorsqu'ils deviennent parents, les carrières des enseignants se différencient sensiblement, avec des interruptions de parcours et un recours au temps partiel plus fréquent chez les femmes que chez les hommes. Ce constat n'est pas propre à l'enseignement, mais il est pertinent ici de noter que ce métier n'échappe pas aux effets différenciés de la parentalité en dépit de sa représentation comme étant un métier offrant les mêmes opportunités de carrière aux hommes et aux femmes.

L'effet de la parentalité sur la différentiation des normes de l'activité professionnelle apparaît aussi plus prononcé en Angleterre qu'en France. En France, parmi la population d'enquête, les mères, y compris lorsqu'elles ont plusieurs enfants en bas âge, adoptent dans l'ensemble des normes d'activité qui restent relativement proches de celles des hommes. Parmi la population d'enquête anglaise, au contraire, la maternité est souvent associée à l'adoption de normes d'activité «spécifiques», caractérisées par des retraits plus ou moins temporaires et partiels du marché avec des interruptions de carrière pouvant aller jusqu'à l'adolescence des enfants et des quotités de travail parfois de l'ordre de $20 \%$ d'un temps plein. Ce n'est pas tant que les enseignantes françaises recourent moins souvent au temps partiel que leurs consœurs britanniques, mais plutôt le fait que ce temps partiel se fait parmi la population d'enquête française sur des durées courtes, avec des quotités de travail élevées et sans toujours renvoyer chez ces femmes à des motifs de «conciliation », qui offre un contraste intéressant entre ces deux pays. Notre étude ne permet de saisir que partiellement ces retraits du marché du travail, puisque seuls des enseignants en poste ont été interrogés. Mais on peut les appréhender à partir des récits diachroniques des enseignantes, ainsi que des récits d'hommes dont la conjointe enseignante a abandonné son activité salariée ou l'a poursuivie à temps partiel après la naissance des enfants.

\section{POLITIQUES PUBLIQUES, CULTURES PROFESSIONNELLES ET ÉGALITÉ HOMMES- FEMMES}

\author{
En France : une forte autonomie spatio- \\ temporelle et des politiques publiques \\ interventionnistes
}

Les entretiens menés confirment l'autonomie dont disposent les enseignants français dans la gestion spatiotemporelle de leur activité. À titre d'exemple, un interviewé utilise l'expression «temps choisi » pour décrire le temps consacré à son activité professionnelle en dehors des heures de cours. Dans un contexte où le pouvoir formel des directions sur les enseignants reste faible en comparaison de ce que l'on observe dans de nombreux pays dont l'Angleterre, les emplois du temps constituent un enjeu considérable. Les directions se montrent généralement accommodantes face aux requêtes, notamment lorsqu'elles renvoient à des motifs familiaux. Une interviewée explique :

Les horaires, on me les aménage, comme je viens de Toulouse et que j'ai des enfants. Globalement, les proviseurs et les proviseurs adjoints essayent toujours de satisfaire les besoins de l'enseignant... Moi, j'ai des conditions de travail très, très bonnes. En ce sens que j'ai un emploi du temps en général qui est très, très bien (Christine, physique appliquée) ${ }^{2}$.

Les volumes horaires apparaissent aussi modulables, ce qui contribuerait à expliquer les variations inter-individuelles importantes dans ce domaine. Les enseignants français de notre population d'enquête consacrent à leur activité professionnelle entre 27 et 60 heures par semaine, une charge horaire qui tend d'ailleurs à diminuer avec l'ancienneté. Combinées avec des politiques publiques de l'articulation vie familiale-vie professionnelle, les caractéristiques spatio-temporelles du métier d'enseignant encourageraient donc une activité continue et à temps plein (ou proche d'un temps plein), y compris chez les mères : 
...quand ils étaient petits, j'ai eu recours à une nourrice, une nourrice agréée. Et puis, quand ils ont commencé à aller à l'école, je les déposais le matin, elle les mettait à l'école... ça n'a pas été un souci. On nous demande quand même de remplir des fiches de vœux, et on essaie, par rapport aux emplois du temps, de commencer à 9 heures et demie, ou bien de finir avant 4 heures et demie. Enfin, ils essaient de nous arranger tant que faire se peut par rapport aux enfants (Marie, anglais/ lettres).

L'ensemble des interviewés de la population d'enquête française s'accordent à décrire leur métier comme favorable à la «conciliation» vie familiale-vie professionnelle. Pour autant, des tensions existent et les ajustements spatio-temporels qui en résultent incombent surtout aux mères. Celles-ci, surtout lorsque les enfants sont en bas âge et a fortiori lorsqu'ils sont nombreux, mettent alors en place des stratégies de rationalisation de leurs activités, gèrent la délégation du domestique à des tiers, voire, pour celles ayant plusieurs enfants en bas âge, ont recours au temps partiel. Les récits qui suivent illustrent le contraste qui existe entre les mères et les pères. Dans le cas du premier extrait, Béatrice explique ainsi comment elle a recours à des stratégies de rationalisation de son temps de travail, notamment lors de la préparation des cours, afin de libérer du temps pour des activités familiales et domestiques. En comparaison, Patrick admet être parfois "contemplatif » lorsqu'il prépare ses cours et apparaît davantage guidé par des préférences personnelles et par le désir de rompre avec la routine du métier :

...je n'ai pas le temps. Je pourrais y passer plus de temps. Là, je fais avec ce que j'ai, je ne retape pas les cours... je suis hyper-efficace... C'est vrai, en une demiheure, je pourrais refaire un cours complètement. Ceci dit, je pourrais y passer 10 fois plus de temps (Béatrice, sciences physiques/mathématiques).

[Je] reprends beaucoup mes cours. Bon, je suis aussi contemplatif. C'est vrai que des fois je pourrais peutêtre y passer moins de temps. J'y vais un petit peu, comment dire, au feeling aussi. C'est-à-dire que je n'ai pas vraiment de stratégie sur l'année... Je peaufine... II y a un truc qui va me cliquer, et je vais repartir sur des nouvelles prépas, je vais refaire tout le chapitre... Mais, c'est aussi un moyen pour moi de ne pas m'ennuyer, de me motiver (Patrick, histoire-géographie).

\section{En Angleterre : des temporalités professionnelles prescriptrices et une faible intervention de l'État}

L'intensification du travail dans les écoles anglaises et ses effets sur la main d'œuvre enseignante constituent des phénomènes bien documentés (Hargreaves, 2000). Les enseignants ayant des responsabilités de management ont des charges d'enseignement «allégées" (les free periods), mais cet allègement ne suffit pas à compenser l'accroissement significatif de la charge de travail associé à l'accès aux fonctions de management. Le management sur la base des résultats, l'obligation de rendre des comptes, le travail en équipe, les missions élargies et le statut moins protecteur constituent autant de facteurs susceptibles de limiter la capacité des enseignants des établissements anglais à moduler leur investissement professionnel en fonction d'autres responsabilités. II apparaît alors peu surprenant que les enseignants anglais de la population d'enquête fassent référence de manière récurrente lors des entretiens aux temporalités professionnelles pour les décrier. La plupart déclarent consacrer environ 50 heures par semaine à leur activité professionnelle (plus de 60 heures pour certains d'entre eux). Les récits des journées de travail mettent en évidence une activité continue tout au long de la journée. Des activités diverses se succèdent à un rythme rapide, en lien avec les responsabilités élargies qui incombent aux enseignants dans ce pays, et ne permettent pas d'accomplir au cours de la journée des activités sans lien direct avec leur emploi, contrairement à ce que nous avons pu observer parmi la population d'enquête française :

J'arrive à 8 heures et quart... Je dirais que de la première à la dernière minute passée dans l'école, je n'arrête pas. Même lorsque je déjeune, je m'occupe d'autres choses (Kathleen, design/technologies alimentaires).

En outre, les temporalités professionnelles apparaissent faiblement négociables avec les directions, en dehors des demandes de travail à temps partiel. La plupart des interviewés déclarent arriver dans l'école entre 7 heures 30 et 8 heures 30 . L'heure de départ varie, mais il est rare qu'ils quittent l'établissement avant 16 heures 30 , surtout lorsqu'ils ont des responsabilités de management. Les récits mettent en évidence une culture professionnelle du "présentiel » et la nature relativement prescrite de ce travail du point de vue de ses conditions spatio-temporelles d'exercice :

Les cours finissent à 3 heures 15 , mais nous avons aussi des activités para-scolaires, ainsi que des compétitions sportives avec d'autres établissements, ce qui peut durer jusqu'à 19 heures 30 le soir... On attend de nous que l'on y prenne part, bien que ce ne soit pas obligatoire, mais on attend de nous que l'on y prenne part (Jack, éducation physique et sportive). 
Les exigences de polyvalence et de disponibilité continue qui caractérisent le travail enseignant en Angleterre ne permettent pas non plus de planifier son emploi du temps de manière très fiable. Comme le résume une interviewée : «ll n'y a pas de journée typique. Cela n'existe pas » (Theresa, français/espagnol). Le temps partiel permet de réduire la charge horaire et, surtout, d'introduire de la flexibilité dans un emploi du temps qui en est fortement dénué. Certains enseignants à temps partiel déclarent ainsi travailler tous les jours de la semaine, le recours au temps partiel permettant d'effectuer ce travail à domicile, là où les enseignants des établissements du second degré français ont la possibilité de concentrer leur emploi du temps sur trois ou quatre jours tout en exerçant leur métier à temps plein. Par ailleurs, si, toujours en Angleterre, ce métier offre de nombreuses possibilités de travail à temps partiel, les postes les plus élevés de la hiérarchie interne du métier ne sont généralement disponibles qu'à temps complet, et le temps partiel est parfois interprété par les directions comme le signe d'un sousinvestissement sur le plan professionnel (Moreau, Osgood \& Halsall, 2008). De manière similaire, si les retours vers le professorat sont possibles après une interruption de carrière, ils ne sont pas assortis de la garantie de retrouver un emploi au même niveau de la hiérarchie. Si ces mesures de flexibilité peuvent favoriser le maintien ou le retour sur le marché du travail, elles peuvent aussi avoir un coût pour ceux (en réalité surtout celles) qui y ont recours du point de vue de la progression de carrière.

Alliées au faible interventionnisme d'État dans l'articulation vie familiale-vie professionnelle, les conditions d'exercice de ce métier, prescriptrices sur un plan spatio-temporel, permettent de comprendre l'impact de la maternité sur les carrières des enseignantes anglaises. On observe ainsi une forte polarisation des carrières féminines, les femmes étant amenées à «choisir » entre activités et production et activités de reproduction, là où leurs homologues françaises sont davantage sur le mode du «cumul». Dans le premier cas de figure, la parentalité (ou, plus précisément, la maternité) est généralement associée avec des parcours à temps partiel (avec une quotité de travail souvent très faible) et avec des interruptions de carrière parfois très longues, afin de gérer les tensions entre les exigences de la vie familiale et celles de la vie professionnelle. En l'absence de soutien suffisant de l'État, le travail de reproduction des mères se substitue donc à celui du personnel des structures scolaires et de soins aux enfants. Des arguments économiques (il n'existe pas d'école maternelle et le coût des structures de garde est fort élevé) et affectifs (la seule forme de travail salarié acceptable pour une mère de jeune enfant est le travail à temps partiel) (European Values Study, 2009) sont souvent évoqués par les interviewés pour justifier le retrait partiel ou total des mères du marché du travail :

[Je voulais travailler à temps partiel] pour avoir plus de temps avec mes enfants. Parce qu'ici il n'y a pas de vrai système pour les petits enfants. II faut trouver des nounous, dans le privé, ensuite les écoles finissent tôt... Pourquoi faire des enfants si c'est pour ne pas les voir? (Sophie, informatique).

Les carrières des hommes enseignants britanniques, comme celles de leurs homologues français, se font généralement de manière continue et à temps complet, y compris chez les pères, qui bénéficient du passage à temps partiel ou du retrait du marché du travail de leur conjointe, parfois elle aussi enseignante. On voit donc ici comment les carrières masculines bénéficient des mesures dites de flexibilité auxquelles ont recours les mères. En se retirant, en partie ou totalement, du marché du travail, celles-ci limitent les incidences de la parentalité sur les carrières masculines en libérant les pères du travail domestique et du care :

\section{Est-ce qu'avoir une famille a ralenti votre carrière?}

... pour moi, non. Mais pour ma femme, oui... Elle a interrompu sa carrière parce qu'elle avait deux enfants... Elle a quitté l'enseignement pendant cinq, six ans, parce qu'elle le voulait et parce que nous pensions que c'était important qu'elle reste à la maison pour s'occuper d'eux. Depuis qu'elle a repris, elle travaille à temps partiel (Neil, mathématiques).

Les enseignantes anglaises privilégiant les activités de production (généralement en mobilité professionnelle ascendante et dont les modalités d'activité sont proches de celles des hommes) sont bien souvent sans enfants. Certaines interviewées font pourtant ici figure d'exception. C'est le cas de Kirsten et de Kathleen, qui vivent toutes les deux en couple avec un conjoint du sexe opposé, ont des enfants encore jeunes et occupent un poste à responsabilités (de middle management dans le cas de Kirsten, de senior management dans le cas de Kathleen). Ces deux interviewées se distinguent de la plupart des autres femmes de la population d'enquête du fait qu'elles décrivent un partage des activités domestiques et familiales plutôt égalitaire (Kathleen) ou en la défaveur de leur conjoint (Kirsten), ce qui n'est jamais le cas parmi la population d'enquête française. Elles évoquent aussi un capital social et culturel plus élevé que celui de leur conjoint (le conjoint de Kathleen est artisan, celui de Kirsten est en reprise d'étude et garde leur enfant au domicile familial, tout en assumant une 
large part des activités domestiques). Alors que cette dernière attend un deuxième enfant, elle souhaite continuer son activité à temps plein et n'envisage pas de revoir à la baisse ses ambitions professionnelles :

...mon compagnon me soutient beaucoup et... m'aide en restant à la maison. Je n'ai pas à m'inquiéter à propos de mon fils et à le mettre dans une crèche, ou des choses de ce genre. Et je pense que cela aide, parce que, lorsque je suis à l'école, je sais qu'il est heureux à la maison et que l'on s'occupe bien de lui (Kirsten, éducation personnelle et sociale/éducation religieuse/psychologie).

Comme le mettent en évidence ces deux cas de figure, dans un contexte où les caractéristiques spatio-temporelles de l'activité salariée sont contraignantes et où l'État peine à investir cette question de l'articulation vie familiale-vie professionnelle, les arrangements égalitaires au sein des couples constituent un déterminant-clé du maintien des enseignantes dans l'emploi à temps plein, a fortiori lorsqu'elles envisagent une mobilité ascendante. Les enseignantes anglaises de notre enquête qui ont des enfants en bas âge et une mobilité ascendante évoquent toujours un conjoint participatif. De la même manière, la présence à proximité de membres de la famille favorise le maintien dans l'emploi à temps plein et la mobilité ascendante des mères. En France, l'implication du conjoint ou un soutien familial apparaissent moins déterminants. Les enseignantes françaises, y compris les mères, sont à même de poursuivre une activité professionnelle dans le cas d'un conjoint peu participatif ou peu disponible ${ }^{3}$ grâce à l'intervention publique dans ce domaine et à la forte autonomie spatio-temporelle qui caractérise l'enseignement français du second degré.

En comparaison de leurs homologues femmes, la parentalité ne semble pas affecter de la même manière les carrières des hommes enseignants anglais. Lorsqu'on les questionne sur ce point, certains évoquent les effets positifs de la parentalité sur la manière dont ils exercent leur métier, voire sur leur carrière. Si, parmi nos enquêtés, deux hommes travaillent à temps partiel, c'est précisément l'absence de responsabilités familiales importantes, et notamment le fait qu'ils n'ont pas d'enfants à charge en bas âge, qui rend le recours au temps partiel possible chez eux, alors même que chez les enseignantes anglaises le temps partiel est justifié par la parentalité :

Si j'avais eu des enfants, j'aurais eu besoin d'une plus grande maison, et si j'avais eu besoin d'une plus grande maison, je n'aurais pas pu être à temps partiel. J'aurais peut-être, contre ma volonté, pris des responsabilités supplémentaires parce que j'aurais eu besoin d'argent, ou alors j'aurais quitté l'enseignement (Andrew, histoire).

À partir de ce qui précède, on voit donc comment, en Angleterre, la parentalité entraîne une polarisation de la situation des hommes et des femmes au regard de leur emploi. Pour les pères, il apparaît difficile d'échapper à un positionnement comme «principal pourvoyeur de ressources » (Lewis, 1992), pour les mères, à l'assignation prioritaire à la sphère domestique. Mais elle entraîne aussi une polarisation des femmes, avec d'une part celles qui, sous l'effet de contraintes structurelles et culturelles, privilégient les activités de reproduction et d'autre part celles qui privilégient les activités de production, là où leurs homologues françaises ont davantage tendance à adopter le régime du «cumul»4.

\section{Des opportunités de carrière similaires pour les enseignants et les enseignantes français?}

Si, en France, un État-providence interventionniste et une forte autonomie de l'activité salariée sur le plan spatio-temporel paraissent favorables à l'égalité professionnelle, les chiffres présentés dans la troisième partie de cet article mettent en évidence la plus forte concentration des hommes dans les postes les plus prestigieux, qui sont aussi souvent les mieux rémunérés. L'agrégation interne, pour un titulaire du CAPES, constitue un concours particulièrement sélectif et est souvent perçue comme la principale voie de promotion. Elle exige une préparation conséquente, et donc du temps, surtout lorsque les enseignants ont affaire à des «petites classes » (ce qui est davantage le cas des femmes, plus présentes en collège). Certes, parmi la population d'enquête, des hommes et des femmes ont réussi ce concours en dépit de responsabilités familiales importantes. Malgré tout, les expériences des pères et des mères diffèrent sensiblement. Les femmes sont davantage susceptibles de renoncer à ce projet du fait de leur situation familiale :

Le fait que je sois à temps partiel, c'est essentiellement lié à une surcharge de travail, donc le fait d'être mère de famille joue effectivement... Je pense que si je n'avais pas d'enfants, je serais à temps complet, je l'aurais probablement passée... Mais là, je n'ai pas pu (Emmanuelle, lettres).

Outre l'agrégation, l'accès à des élèves ayant un niveau élevé dans la discipline (par exemple des classes de lycée ou de l'enseignement post-baccalauréat, notamment lorsqu'il s'agit de sections à examen avec un fort coefficient dans la discipline enseignée) est parfois perçu comme une évolution sur le plan professionnel, du fait 
d'identités professionnelles dont l'expertise disciplinaire constitue le ressort principal. L'enseignement à ces classes se traduit bien souvent par un accroissement de la charge de travail. En effet, outre la préparation de nouveaux cours, cela requiert aussi parfois une remise à niveau dans la discipline :

Là, je suis en lycée. Mais, si tu veux, les années que j'ai passées en collège... La première année, j'ai dû travailler quand même pas mal... Mais les six dernières années, mes cours étaient prêts, donc à part mes corrections, je n'avais rien. II y avait certains week-ends où je n'ouvrais pas mon sac. C'est pour ça que je suis passée d'un extrême à l'autre (Fabienne, anglais).

Les mères apparaissent plus hésitantes à demander un lycée, non pas par préférence personnelle pour les petites classes, mais parce que l'accroissement des temps de travail que cela exigerait se heurterait à leur investissement dans la sphère domestique (CacouaultBitaud, 2007). Cela amène certaines à repousser ce projet, ce qui revient parfois à l'abandonner puisque, au fil du temps, les enseignants des petites classes éprouvent souvent un sentiment de déqualification du point de vue de leur expertise disciplinaire :

Je veux rester prof au collège. Enfin, ça ne veut pas dire qu'un jour je ne demanderai pas le lycée de [ville]. Mais peut-être quand [mon fils] aura un peu grandi, quand j'aurai plus de temps... Et puis avec le temps, maintenant, c'est peut-être idiot, mais je ne me verrais plus retourner au lycée, aussi pour des raisons liées à ma discipline. Enfin, c'est vrai qu'il faudrait que je retravaille énormément, et je ne m'en sens pas le temps maintenant avec le petit (Élise, mathématiques).

L'implication dans des projets spécifiques au niveau de l'établissement, du rectorat ou du ministère constitue aussi pour certains enseignants une forme de valorisation des compétences professionnelles. Parmi les enseignantes, certaines ont connu une mobilité géographique importante (Jaboin, 2003) liée aux mutations professionnelles de leur conjoint et au primat bien souvent accordé à la carrière de celui-ci. Or, l'accès à ce type de responsabilité nécessite de s'être "fait repérer » par les inspections ou les directions, ce que ne favorisent pas les changements fréquents d'établissement. Par ailleurs, les classes à examen avec un coefficient élevé dans la discipline sont parfois "réservées" aux enseignants ayant une forte ancienneté dans l'établissement, comme le notent certains interviewés.

Or, du fait de demandes de mutations répétées afin d'accommoder la carrière de leur conjoint, certaines enseignantes ne peuvent prétendre qu'à des postes de Titulaires sur zone de remplacement (TZR), qui limitent leur capacité à s'investir dans des projets locaux puisque ces postes ne sont assortis d'aucune garantie de pouvoir rester dans l'établissement l'année suivante. Le statut de TZR ou des mobilités répétées visant à accommoder la carrière du conjoint et non sa carrière propre peuvent donc freiner l'investissement dans ces projets, l'accès à des classes ayant un niveau avancé dans la discipline, mais aussi l'exercice de responsabilités du type professeur principal :

...comme je suis toujours TZR, je ne suis jamais sûre d'être dans le même lycée, et c'est difficile de m'investir dans un projet sur plus d'une année, à part des projets avec les élèves à l'année... (Béatrice, sciences physiques/mathématiques).

...en tant que TZR, vous ne pouvez pas demander [à être professeur principal]. Parce que vous arrivez et vous faites les choses qui restent... (Sabine, économiegestion).

Ce type de parcours n'a rien d'exceptionnel en France (Jaboin, 2003). Il caractérise plusieurs de nos interviewées et est aussi lié aux alliances matrimoniales des enseignantes françaises, bien que les enseignantes qui "suivent » leur conjoint n'aient pas toujours des revenus inférieurs. Ces mutations répétées peuvent aussi avoir des effets significatifs sur les vies personnelles des enseignants, notamment dans le cas de reconversions professionnelles tardives (un phénomène qui, dans notre étude, caractérise davantage les femmes), lorsque les interviewés ne parviennent pas à obtenir un poste dans la ville, voire dans l'académie où leur conjoint a obtenu une mutation.

\section{CONCLUSION}

Si l'enseignement du second degré est généralement considéré comme un métier offrant des opportunités similaires aux hommes et aux femmes, les analyses qui précèdent questionnent cette représentation. L'analyse comparative France-Angleterre, s'appuyant sur des données statistiques et sur un corpus d'entretiens menés en France et en Angleterre auprès d'enseignants des établissements du second degré, met au jour la différentiation sexuée des carrières enseignantes dans les deux pays. Mais la perspective comparative retenue permet aussi de remettre en cause une vision universelle et «naturaliste» du genre (Guillaumin, 1992). En effet, si, dans les deux pays considérés, les femmes sont sous-représentées dans les 
segments du marché du travail enseignant associés au capital économique et symbolique le plus élevé, on observe aussi une certaine variabilité des formes de cette différentiation sexuée, qui apparaît liée aux politiques publiques et aux cultures professionnelles qui prévalent dans chaque contexte.

Ainsi, dans le contexte français, l'intervention des politiques publiques dans l'articulation vie familiale-vie professionnelle, combinée à une forte autonomie des enseignants du second degré sur le plan spatio-temporel, favorise l'alignement des normes d'activité des hommes et des femmes, y compris chez les mères. En Angleterre, le faible interventionnisme de l'État dans ce domaine, allié à des conditions d'exercice de ce métier plus contraignantes sur un plan spatio-temporel, favorise la polarisation des carrières féminines et masculines lorsque les enseignants deviennent parents, mais aussi la polarisation entre carrières féminines, les enseignantes anglaises étant alors amenées à «choisir » (un choix en réalité fortement contraint d'un point de vue structurel et culturel) entre activités dites de production et celles dites de reproduction. Mais si cet article évoque principalement les inégalités professionnelles, on notera qu'en France les usages genrés de la flexibilité spatio-temporelle de ce métier, alliés à l'hypergamie des enseignantes françaises, dont le conjoint occupe bien souvent un emploi plus rémunérateur et plus prestigieux (Cacouault-Bitaud, 2007), favorisent des arrangements domestiques inégalitaires (Moreau, 2011a). Sans revenir ici de manière détaillée sur ce point, on peut lire dans ce constat une invitation à regarder de manière plus détaillée ce qui se passe du côté du care dans des travaux futurs. De manière plus générale, les analyses proposées montrent que, plutôt que d'être liée à des préférences personnelles, à des styles de vie (Hakim, 2000) ou encore à la socialisation différenciée des hommes et des femmes (Jaboin, 2003), la concentration des femmes dans les postes les moins élevés de la hiérarchie interne du métier a à voir avec les contraintes structurelles et culturelles propres à chaque contexte national, en matière d'intervention publique ou de conditions de travail, de recrutement et de promotion propres à chaque catégorie d'emploi.

Marie-Pierre Moreau Marie-Pierre.Moreau@roehampton.ac.uk University of Roehampton

\section{NOTES}

1 Le taux de féminisation varie aussi en fonction de la discipline enseignée (Department for Education, 2013; Ministère de l'Éducation nationale, 2013). Cet aspect ne fait cependant pas l'objet de cet article, qui porte avant tout sur la ségrégation verticale des carrières enseignantes. Les données quantitatives et qualitatives disponibles ne permettent pas de tirer de conclusions quant à un éventuel lien entre la discipline enseignée et la progression de carrière. Rappelons par ailleurs que, chez les enseignants des établissements anglais du second degré, la bivalence est de rigueur, que les disciplines enseignées changent en fonction des besoins des établissements et qu'elles ne sont pas toujours celles étudiées à l'université, surtout dans les disciplines déficitaires (Hutchings, 2011), rendant ainsi l'effet de la discipline sur les carrières difficiles à saisir.
2 Tous les prénoms sont des pseudonymes.

3 Ce qui est souvent le cas parmi les enseignantes françaises, qui sont davantage susceptibles que leurs homologues anglaises d'être en couple avec un homme occupant une profession plus prestigieuse et attirant des revenus plus élevés que la leur (Moreau, 2011a).

4 On note d'ailleurs que, parmi les enseignantes françaises interrogées, seules deux n'ont pas d'enfant (l'une d'entre elles évoquant d'ailleurs un projet familial), alors que ce cas de figure concerne six enseignantes britanniques.

\section{BIBLIOGRAPHIE}

BUSCATTO M. \& MARRY C. (2009). «Le plafond de verre dans tous ses éclats. La féminisation des professions supérieures au Xxe siècle». Sociologie du travail, vol.51, n², p. 170-182.

CACOUAULT M. (1987). «Prof, c'est bien... pour une femme». Le Mouvement social, n¹40, p. 107-119.

CACOUAULT M. (2003). «La sociologie de l'éducation et les enseignants... cherchez la femme». In J. Laufer, C. Marry \& M. Maruani (dir.), Le travail du genre: les sciences sociales du travail à l'épreuve des différences de sexe. Paris : La Découverte.
CACOUAULT-BITAUD M. (2007). Professeurs mais femmes. Carrières et vies privées des enseignantes du secondaire au $x x^{e}$ siècle. Paris : La Découverte.

CHADEAU A. \& FOUQUET A. (1981). «Peut-on mesurer le travail domestique? ". Économie et statistique, no136, p.29-42.

CHAN A. K. W., NGAI G.S. K. \& CHOI P. K. (2014). «Contextualizing the career pathways of women principals in Hong Kong: A critical examination ". Compare : A Journal of Comparative and International Education. En ligne : <http://www. tandfonline.com/doi/full/10.1080/03057925.2014.884919\#. VBF76q6hFIA> (consulté le 12 septembre 2014). 
COLEMAN M. (2002). Women as headteachers: striking a balance. Stoke-on-Trent: Trentham Books.

COLLECTIF (1984). Le sexe du travail. Structures familiales et système productif. Grenoble : Presses universitaires de Grenoble.

CROMPTON R. (dir.) (1999). Restructuring gender relations and employment: the decline of the male breadwinner. Oxford: Oxford University Press.

DAUNE-RICHARD A.-M. (1999). «La notion de référentiel appliquée à la garde des jeunes enfants : une comparaison France-Suède». Recherches et prévisions, n56, p. 33-47.

DELPHY C. (1993). «Rethinking sex and gender ». Women's Studies International Forum, no16, p. 1-9.

DEPARTMENT FOR EDUCATION (2013). School workforce in England. Londres : TSO.

ESPING-ANDERSEN G. (1990). The three worlds of welfare capitalism. Cambridge : Polity Press.

EUROPEAN VALUES STUDY (2009). En ligne : <http://www.europeanvaluesstudy.eu/evs/surveys/survey-2008.html> (consulté le 28 mars 2011).

FAUCHER-KING F. \& LE GALÈS P. (2010). Les Gouvernements New Labour. Le bilan de Tony Blair et Gordon Brown. Paris : Presses de Sciences Po.

FAVE-BONNET M.-F. (1999). "Les femmes universitaires en France : une féminisation... mais différenciée». In N. Le Feuvre, M. Membrado \& A. Rieu (dir.), Les femmes et l'université en Méditerranée. Toulouse : Presses universitaires du Mirail.

FORTINO S. (2002). La mixité au travail. Paris : La Dispute.

FOUQUET A. \& LAUFER J. (2001). «Les cadres à l'épreuve de la féminisation ». In P. Bouffartigue (dir.), Cadres : la grande rupture. Paris : La Découverte.

GLENNERSTER H. (1991). «Quasi-markets for education? ». Economic Journal, no101, p.1268-1276.

GREGORY A. \& WINDEBANK J. (2000). Women's work in Britain and France: practice, theory and policy. Londres : Palgrave MacMillan.

GUILLAUMIN C. (1992). Sexe, race et pratique du pouvoir. L'idée de nature. Paris : Côté-Femmes.

HAKIM C. (2000). Work-Lifestyle Choices in the 21st Century: Preference Theory. Oxford : Oxford University Press.

HARGREAVES A. (2000). «Four ages of professionalism and professional learning Teachers and Teaching ». Teachers and Teaching: Theories and Practice, n॰6, p. 151-182.

HIRATA H., LABORIE F., LE DOARE H. \& SENOTIER D. (dir.) (2000). Dictionnaire critique du féminisme. Paris : PUF.

HUTCHINGS M. (2011). What impact does the wider economic situation have on teachers' career decisions? A literature review (Research Report DFE-RR136). Londres : Department for Education.

HUTCHINGS M., SMART S., JAMES K. \& WILLIAMS K. (2006). General Teaching Council: Survey of Teachers 2006. Londres : Institute for Policy Studies in Education, London Metropolitan University.
INSEE (2011). France, portrait social. Paris : INSEE.

JABOIN Y. (2003). Le prof dans tous ses états : féminin ou masculin, public ou privé. Paris : Fabert.

LALLEMENT M. \& SPURK J. (dir.) (2003). Stratégies de la comparaison internationale. Paris : CNRS.

LE FEUVRE N. (dir.) (2002). Le genre : de la catégorisation du sexe. Toulouse : UTINAM-L'Harmattan.

LE FEUVRE N. (2008). «La pluralité des modèles de féminisation des professions supérieures en France et en Grande-Bretagne ». In H. Hirata, M. R. Lombardi \& M. Maruani (dir.), Travail et genre : regards croisés France-Europe-Amérique latine. Paris : La Découverte.

LE FEUVRE N., LAPEYRE N., CACOUAULT M. \& PICOT G. (2003). La féminisation des professions libérales : l'exemple des professions médicales et juridiques. Paris : Ministère de l'Emploi et de la Solidarité, Service des droits des femmes et de l'égalité, étude no 75 .

LÉGER A. (1983). Les enseignants du secondaire. Paris : PUF.

LEWIS J. (1992). «Gender and the development of welfare regimes ». Journal of European Social Policy, vol. 2, n³, p. 159-173.

MAHONY P. \& HEXTALL I. (2000). Reconstructing teaching: Standards, performance and accountability. Londres : Routledge Falmer.

MALET R. \& BRISARD E. (dir.) (2005). Modernisation de l'école et contextes culturels : des politiques aux pratiques en France et en Grande-Bretagne. Paris : L'Harmattan.

MARTIN J. (1998). "Le rôle de l'État dans la construction sociale de la relation mère-enfant ». In A. Fine, À chacun sa famille. Toulouse : Presses universitaires du Sud.

MARRY C. (1998). "La comparaison France-Allemagne à l'épreuve des femmes". In M. Maruani (dir.), Les nouvelles frontières de l'inégalité : hommes-femmes sur le marché du travail. Paris : La Découverte.

MAURICE M. (1989). «Méthode comparative et analyse sociétale : les implications théoriques des comparaisons internationales ". Sociologie du travail, n², p. 175-191.

MAURICE M., SELLIER F. \& SILVESTRE J. (1982). Politique d'éducation et organisation industrielle en France et en Allemagne : essai d'analyse sociétale. Paris : PUF.

MAURICE M., SELLIER F. \& SILVESTRE J. (1992). «Analyse sociétale et cultures nationales : réponse à Philippe d'Iribarne ». Revue française de sociologie, vol. 33, n॰1, p. 75-86.

MINISTÈRE DE L'ÉDUCATION NATIONALE (2013). Repères et références statistiques. Paris : Ministère de l'Éducation nationale.

MOREAU M.-P. (2011a). Les enseignants et le genre : les inégalités hommes-femmes dans l'enseignement du second degré en France et en Angleterre. Paris : PUF.

MOREAU M.-P. (2011b). «La différentiation sexuée des carrières enseignantes en France et en Angleterre : un impensé sociologique et politique». In M. Cacouault \& F. Charles, Quelle mixité dans les formations et les groupes professionnels? Enquêtes sur les frontières et le mélange des genres. Paris : L'Harmattan. 
MOREAU M.-P. (2013). «Becoming a secondary school teacher in England and France: the societal construction of career "choice" ". Compare: A Journal of Comparative and International Education. En ligne : <http://www.tandfonline.com/ doi/full/10.1080/03057925.2013.876310\#.U570P65ORIA> (consulté le 11 septembre 2014).

MOREAU M.-P. (dir.) (2014). Inequalities in the Teaching Profession: A global perspective. Londres; New York : Palgrave McMillan.

MOREAU M.-P., OSGOOD J. \& HALSALL A. (2008). «Equal opportunities policies in english schools: towards greater gender equality in the teaching workforce? ". Gender, Work and Organization, vol. 15, n॰6, p.553-578.

MOREAU M.-P., OSGOOD J. \& HALSALL A. (2007). «Making sense of the glass ceiling: an exploration of women teachers' discourses ". Gender \& Education, vol. 19, n², p. 237-253.

O'REILLY J. (2000). «Is it time to gender the societal effects? ». In M. Maurice \& A. Sorge, Embedding organizations: societal analysis of actors, organizations and socioeconomic context. Amsterdam : John Benjamins.

RAVEAUD M. (2006). De l'enfant au citoyen. Paris, PUF.

ROBSON C. (1993). Real World Research: A resource for social scientists and practitioner-researchers. Oxford : Blackwell.

WILLIAMS C. (1992). "The glass escalator: Hidden advantages for men in the "female professions" ". Social Problems, n०39, p. 253-67. 


\section{ANNEXE : DESCRIPTION DE LA POPULATION D'ENQUÊTE (ENTRETIENS PRINCIPAUX)}

\section{Tableau 1. Population d'enquête en France}

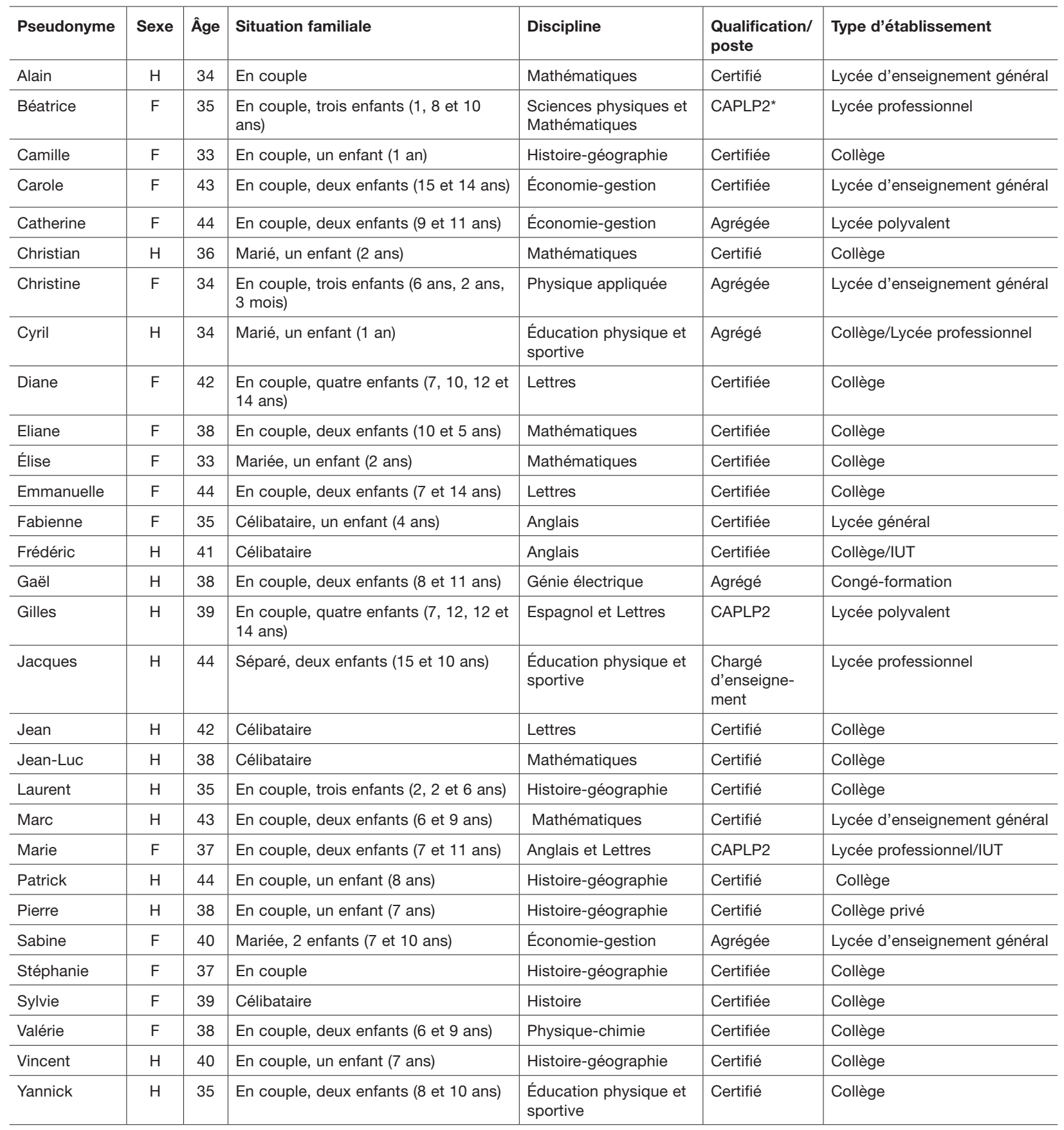

Note : * : Certificat d'aptitude au professorat de lycée professionnel 
Tableau 2. Population d'enquête en Angleterre

\begin{tabular}{|c|c|c|c|c|c|c|}
\hline Pseudonyme & Sexe & Âge & Situation familiale & Discipline enseignée & $\begin{array}{l}\text { Qualification/ } \\
\text { poste }\end{array}$ & Type d'établissement \\
\hline Alex & $\mathrm{H}$ & 50 & $\begin{array}{l}\text { En couple, quatre enfants ( } 12 \text {, } \\
17,21 \text { et } 22 \text { ans) }\end{array}$ & Mathématiques & $\begin{array}{l}\text { Classroom } \\
\text { teacher }\end{array}$ & $\begin{array}{l}\text { Comprehensive girls' } \\
\text { school }\end{array}$ \\
\hline Alistair & $\mathrm{H}$ & 43 & $\begin{array}{l}\text { En couple, deux enfants (sco- } \\
\text { larisés; âge non divulgué) }\end{array}$ & Géographie & Deputy Head & $\begin{array}{l}\text { Comprehensive school } \\
\text { with sixth-form }\end{array}$ \\
\hline Andrew & $\mathrm{H}$ & 44 & En couple & Histoire & $\begin{array}{l}\text { Classroom } \\
\text { teacher }\end{array}$ & $\begin{array}{l}\text { Comprehensive girls' } \\
\text { school }\end{array}$ \\
\hline Ashton & $\mathrm{H}$ & 45 & En couple, un enfant (10 ans) & Science & Head of Year & $\begin{array}{l}\text { Comprehensive school } \\
\text { with sixth-form }\end{array}$ \\
\hline Carl & $\mathrm{H}$ & 32 & En couple & Science et Français & $\begin{array}{l}\text { Deputy Head } \\
\text { of Faculty }\end{array}$ & $\begin{array}{l}\text { Comprehensive school } \\
\text { with sixth-form }\end{array}$ \\
\hline Carlyn & $\mathrm{F}$ & 40 & $\begin{array}{l}\text { En couple, deux enfants ( } 9 \text { et } \\
14 \text { ans) }\end{array}$ & $\begin{array}{l}\text { Éducation physique et sportive et } \\
\text { Éducation personnelle, sociale et à } \\
\text { la santé }\end{array}$ & $\begin{array}{l}\text { Classroom } \\
\text { teacher }\end{array}$ & $\begin{array}{l}\text { Comprehensive school } \\
\text { with sixth-form }\end{array}$ \\
\hline Christopher & $\mathrm{H}$ & 39 & Célibataire & Mathématiques & $\begin{array}{l}\text { Deputy } \\
\text { headteacher }\end{array}$ & $\begin{array}{l}\text { Comprehensive girls' } \\
\text { school }\end{array}$ \\
\hline Clare & $\mathrm{F}$ & 39 & En couple, un enfant ( 2 ans) & Lettres & $\begin{array}{l}\text { Head of } \\
\text { subject }\end{array}$ & $\begin{array}{l}\text { Comprehensive girls' } \\
\text { school }\end{array}$ \\
\hline Cynthia & $\mathrm{F}$ & 33 & En couple & Lettres & $\begin{array}{l}\text { Deputy head } \\
\text { of Subject }\end{array}$ & $\begin{array}{l}\text { Comprehensive girls' } \\
\text { school }\end{array}$ \\
\hline Emma & $\mathrm{F}$ & 46 & $\begin{array}{l}\text { En couple, deux enfants (13 et } \\
16 \text { ans) }\end{array}$ & Lettres & $\begin{array}{l}\text { Classroom } \\
\text { teacher }\end{array}$ & $\begin{array}{l}\text { Comprehensive girls' } \\
\text { school }\end{array}$ \\
\hline Esther & $\mathrm{F}$ & 36 & Célibataire & Espagnol et Français & $\begin{array}{l}\text { Clasroom } \\
\text { teacher } \\
\text { Head of year }\end{array}$ & $\begin{array}{l}\text { Comprehensive school } \\
\text { with sixth-form }\end{array}$ \\
\hline Gemma & $\mathrm{F}$ & 36 & En couple & Français et Espagnol & $\begin{array}{l}\text { Classroom } \\
\text { teacher }\end{array}$ & $\begin{array}{l}\text { Comprehensive school } \\
\text { with sixth-form }\end{array}$ \\
\hline Heather & $\mathrm{F}$ & 40 & $\begin{array}{l}\text { Célibataire, deux enfants ( } 3 \text { et } \\
7 \text { ans) }\end{array}$ & Lettres & Headteacher & $\begin{array}{l}\text { Comprehensive school } \\
\text { with sixth-form }\end{array}$ \\
\hline Henry & $\mathrm{H}$ & 37 & $\begin{array}{l}\text { En couple, deux enfants ( } 3 \text { et } \\
6 \text { ans) }\end{array}$ & $\begin{array}{l}\text { Mathématiques et Éducation phy- } \\
\text { sique et sportive }\end{array}$ & $\begin{array}{l}\text { Head of } \\
\text { Subject/ } \\
\text { Director of } \\
\text { Learning }\end{array}$ & $\begin{array}{l}\text { Comprehensive school } \\
\text { with sixth-form }\end{array}$ \\
\hline Jack & $\mathrm{H}$ & 36 & $\begin{array}{l}\text { En couple, trois enfants }(1,4 \\
\text { et } 8 \text { ans) }\end{array}$ & Éducation physique et sportive & $\begin{array}{l}\text { Head of } \\
\text { Subject }\end{array}$ & $\begin{array}{l}\text { Comprehensive school } \\
\text { with sixth-form }\end{array}$ \\
\hline John & $\mathrm{H}$ & 38 & Célibataire & Cinéma et Études des médias & $\begin{array}{l}\text { Head of } \\
\text { Faculty }\end{array}$ & Sixth-form college \\
\hline Kathleen & $\mathrm{F}$ & 45 & $\begin{array}{l}\text { En couple, deux enfants (11 et } \\
16 \text { ans) }\end{array}$ & Design et Technologies alimentaires & $\begin{array}{l}\text { Head of sixth- } \\
\text { form } \\
\text { consortium }\end{array}$ & $\begin{array}{l}\text { Comprehensive school } \\
\text { with sixth-form }\end{array}$ \\
\hline Kathy & $\mathrm{F}$ & 44 & En couple & Mathématiques & Head of Year & $\begin{array}{l}\text { Comprehensive school } \\
\text { with sixth-form }\end{array}$ \\
\hline Kimberley & $\mathrm{F}$ & 37 & Vit avec sa mère et sa sœur & Science & $\begin{array}{l}\text { Classroom } \\
\text { teacher }\end{array}$ & Sixth-form college \\
\hline
\end{tabular}




\begin{tabular}{|c|c|c|c|c|c|c|}
\hline Kirsten & $\mathrm{F}$ & 36 & En couple, un enfant ( 2 ans) & $\begin{array}{l}\text { Éducation personnelle, sociale et à } \\
\text { la santé, Éducation religieuse et } \\
\text { Psychologie }\end{array}$ & $\begin{array}{l}\text { Deputy head } \\
\text { of year }\end{array}$ & $\begin{array}{l}\text { Comprehensive school } \\
\text { with sixth-form }\end{array}$ \\
\hline Nathan & $\mathrm{H}$ & 44 & $\begin{array}{l}\text { En couple, } 2 \text { enfants (âge non } \\
\text { divulgué) }\end{array}$ & Science & Headteacher & $\begin{array}{l}\text { Secondary school (girls' } \\
\text { school) }\end{array}$ \\
\hline Paula & $\mathrm{F}$ & 36 & $\begin{array}{l}\text { En couple, deux enfants ( } 5 \text { et } \\
7 \text { ans) }\end{array}$ & Informatique & $\begin{array}{l}\text { Classroom } \\
\text { teacher }\end{array}$ & Sixth-form college \\
\hline Peter & $\mathrm{H}$ & 37 & $\begin{array}{l}\text { En couple, deux enfants ( } 3 \\
\text { ans et un nouveau-né) }\end{array}$ & $\begin{array}{l}\text { Mathématiques et Éducation phy- } \\
\text { sique et sportive }\end{array}$ & Head of Year & $\begin{array}{l}\text { Comprehensive school } \\
\text { with sixth-form }\end{array}$ \\
\hline Sophie & $\mathrm{F}$ & 45 & $\begin{array}{l}\text { En couple, deux enfants (13 et } \\
16 \text { ans) }\end{array}$ & Informatique & $\begin{array}{l}\text { Classroom } \\
\text { teacher }\end{array}$ & Sixth-form college \\
\hline Theresa & $\mathrm{F}$ & 42 & En couple & Français et Espagnol & Assistant head & $\begin{array}{l}\text { Comprehensive school } \\
\text { with sixth-form }\end{array}$ \\
\hline Thomas & $\mathrm{H}$ & 48 & En couple, un enfant ( 3 ans) & $\begin{array}{l}\text { Étude des Médias et Études de } \\
\text { commerce }\end{array}$ & $\begin{array}{l}\text { Head of } \\
\text { Subject }\end{array}$ & $\begin{array}{l}\text { Comprehensive girls' } \\
\text { school }\end{array}$ \\
\hline Timothy & $\mathrm{H}$ & 45 & $\begin{array}{l}\text { Divorcé, trois enfants ( } 1 \text { en } \\
\text { garde alternée, } 9 \text { ans; } 18 \text { et } \\
23 \text { ans) }\end{array}$ & Économie & Headteacher & Secondary school \\
\hline
\end{tabular}

